\title{
Some Star Complements for the Second Largest Eigenvalue of a Graph
}

\author{
Zoran Stanić * \\ Faculty of Mathematics, University of Belgrade, Studentski trg 16, 11000 Belgrade, Serbia
}

Received 19 July 2007, accepted 11 September 2008, published online 14 October 2008

\begin{abstract}
The star complement technique is a spectral tool recently developed for constructing some bigger graphs from their smaller parts, called star complements. Often the implementation of this technique requires the use of computers. Therefore, we develop an SCL (star complement library) - a set of programs providing quick implementation of those technique. Here, we present the facilities of SCL. In further, we determine some star complements for 1 or $\frac{\sqrt{5}-1}{2}$ as the second largest eigenvalue of a graph. Finally, using the SCL, we consider the maximal extensions of the star complements obtained.
\end{abstract}

Keywords: Adjacency matrix, graph eigenvalues, star complement, divisor concept, mathematical software.

Math. Subj. Class.: 05C50, 68735

\section{Introduction}

We consider only simple graphs, that is finite, undirected graphs without loops or multiple edges. If $G$ is such a graph with vertex set $V(G)=\{1,2, \ldots, n\}$, the adjacency matrix of $G$ is $n \times n$ matrix $A=A(G)=\left(a_{i j}\right)$, where $a_{i j}=1$ if there is an edge between the vertices $i$ and $j$, and 0 otherwise. The eigenvalues of $G$, denoted by $\lambda_{1} \geq \lambda_{2} \geq \cdots \geq \lambda_{n}$, are just the eigenvalues of $A$. Additionally, for connected graphs $\lambda_{1}>\lambda_{2}$ holds. The characteristic polynomial of $G$ is the characteristic polynomial of its adjacency matrix given by $P_{G}(\lambda)=\operatorname{det}(\lambda I-A)$. For more details on graph spectra, see [3].

Our main goal is to introduce the facilities of freely available software SCL and to identify some graphs having the simple structure which can be star complements for some specific second largest eigenvalues. The graphs whose second largest eigenvalue is (less than or) equal

\footnotetext{
${ }^{*}$ Research is partially supported by Serbian Ministry of Science, Project 144032D.

E-mail address: zstanic@matf.bg.ac.yu (Zoran Stanić)
} 
to 1 or $\frac{\sqrt{5}-1}{2}$ are intensively studied in the literature and they are not completely determined so far. Therefore, the maximal extensions which are determined in this paper can be viewed as a result from that context as well. Additionally, the maximal extensions obtained have small number of distinct eigenvalues, and they can be interesting in some future research.

In Section 2, we give the main definitions, fix some notation and mention some results from the literature in order to make the paper more self-contained. In Section 3, we describe the software SCL which implements an algorithm (originally described in [10]) for constructing the maximal graphs with a specified star complement for a specified eigenvalue. In the remaining two sections, we consider some star complements for the second largest eigenvalue of a graph. First, we complete earlier calculation from [14] which determine the unicyclic star complements for 1 as the second largest eigenvalue. In further we determine all cocktail party graphs and the graphs with up to five vertices which can be star complements for the same eigenvalue. Finally, we determine all trees, unicyclic and bicyclic graphs which can be star complements for $\frac{\sqrt{5}-1}{2}$ as the second largest eigenvalue. Using the SCL, we consider the maximal extensions for all star complements obtained.

\section{Preliminaries}

First, we give a short description of the star complement technique. If $\mu$ is an eigenvalue of $G$ of multiplicity $k$, then a star set for $\mu$ in $G$ is a set $X$ of $k$ vertices taken from $G$ such that $\mu$ is not an eigenvalue of $G-X$. The graph $H=G-X$ is then called a star complement for $\mu$ in $G$ (or a $\mu$-basic subgraph of $G$ in [10]). Star sets and star complements exist for any eigenvalue and any graph; they need not be unique. In addition, for any eigenvalue $\mu$ of a connected graph $G, G$ has a connected star complement for $\mu$ (see [10]). The $H$-neighborhood of an arbitrary vertex in $X$ contains all its neighbors from $H$. The $H$-neighborhoods of vertices in $X$ can be shown to be non-empty and distinct, provided that $\mu \notin\{-1,0\}$ (see [6], Chapter 7). If $t=\left|V_{H}\right|$, then $|X| \leq\left(\begin{array}{l}t \\ 2\end{array}\right)$ (see [1]) and this bound is best possible.

It can be proved that if $Y$ is a proper subset of $X$ then $X-Y$ is a star set for $\mu$ in $G-Y$, and therefore $H$ is a star complement for $\mu$ in $G-Y$. If $G$ has star complement $H$ for $\mu$, and $G$ is not a proper induced subgraph of some other graph with star complement $H$ for $\mu$, then $G$ is a maximal graph with star complement $H$ for $\mu$, or it is an $H$-maximal graph for $\mu$. By the above remarks, there are only finitely many such maximal graphs, provided $\mu \notin\{-1,0\}$. In general, there will be only several maximal graphs, possibly of different orders, but sometimes there is a unique maximal graph (if so, this graph is characterized by its star complement for $\mu$ ).

We now mention some results from the literature regarding the star complement technique (they are taken from [5], [6] and [7]).

The following result is known as the Reconstruction Theorem (see, for example, [6], Theorems 7.4.1 and 7.4.4).

Theorem 1. Let $G$ be a graph with adjacency matrix

$$
\left(\begin{array}{cc}
A_{X} & B^{T} \\
B & C
\end{array}\right),
$$

where $A_{X}$ is the adjacency matrix of the subgraph induced by the vertex set $X$. Then $X$ is a star set for $\mu$ if and only if $\mu$ is not an eigenvalue of $C$ and $\mu I-A_{X}=B^{T}(\mu I-C)^{-1} B$. 
From the above, we see that if $\mu, C$ and $B$ are fixed then $A_{X}$ is uniquely determined. In other words, given the eigenvalue $\mu$, a star complement $H$ for $\mu$, and the $H$-neighborhoods of the vertices in the star set $X$, the graph $G$ is uniquely determined. In the light of these facts, we may next ask to what extent $G$ is determined only by $H$ and $\mu$. Having in mind the observation above, it is sufficient to consider graphs $G$ which are $H$-maximal for $\mu$.

Following [2], [14] and [13], we will now fix some notation and terminology. Given a graph $H$, a subset $U$ of $V(H)$ and a vertex $u$ not in $V(H)$, denote by $H(U)$ the graph obtained from $H$ by joining $u$ to all vertices of $U$. We will say that $u(U, H(U))$ is a good vertex (resp. good set, good extension) for $\mu$ and $H$, if $\mu$ is an eigenvalue of $H(U)$ but is not an eigenvalue of $H$. By Theorem 1, a vertex $u$ and a subset $U$ are good if and only if $\mathbf{b}_{u}^{T}(\mu I-C)^{-1} \mathbf{b}_{u}=\mu$, where $\mathbf{b}_{u}$ is the characteristic vector of $U$ (with respect to $V(H)$ ) and $C$ is the adjacency matrix of $H$. Assume now that $U_{1}$ and $U_{2}$ are not necessarily good sets corresponding to vertices $u_{1}$ and $u_{2}$, respectively. Let $H\left(U_{1}, U_{2} ; 0\right)$ and $H\left(U_{1}, U_{2} ; 1\right)$ be the graphs obtained by adding to $H$ both vertices, $u_{1}$ and $u_{2}$, so that they are non-adjacent in the former graph, while adjacent in the latter graph. We say that $u_{1}$ and $u_{2}$ are good partners and that $U_{1}$ and $U_{2}$ are compatible sets if $\mu$ is an eigenvalue of multiplicity two either in $H\left(U_{1}, U_{2} ; 0\right)$ or in $H\left(U_{1}, U_{2} ; 1\right)$. (Note, if $\mu \notin\{-1,0\}$, any good set is non-empty, any two of them if corresponding to compatible sets are distinct; see [6], cf. Proposition 7.6.2.) By Theorem 1, two vertices $u_{1}$ and $u_{2}$ are good partners (or two sets $U_{1}$ and $U_{2}$ are compatible) if and only if $\mathbf{b}_{u_{1}}^{T}(\mu I-C)^{-1} \mathbf{b}_{u_{2}} \in\{-1,0\}$, where $\mathbf{b}_{u_{1}}$ and $\mathbf{b}_{u_{2}}$ are defined as above. In addition, it follows (again by Theorem 1 ) that any vertex set $X$ in which all vertices are good, both individually and in pairs, gives rise to a good extension, say $G$, in which $X$ can be viewed as a star set for $\mu$, while $H$ as the corresponding star complement.

The above considerations shows us how we can introduce a technique, also called a star complement technique, for finding (or constructing) graphs with certain spectral properties. In this context the graphs we are interested in should have some prescribed eigenvalue usually of a very large multiplicity. If $G$ is a graph in which $\mu$ is an eigenvalue of multiplicity $k>1$, then $G$ is a good ( $k$-vertex) extension of some of its star complements, say $H$ (in particular, $G$ is $H$-maximal for $\mu$ ). The star complement technique consists of the following: In order to find $H$-maximal graphs for $\mu(\neq-1,0)$, we form an extendability graph whose vertices are good vertices for $\mu$ and $H$, and add an edge between two good vertices whenever they are good partners. Now it is easy to see that the search for maximal extensions is reduced to the search for maximal cliques in the extendability graph (see, for example, [10], [5] or [7]). Of course, among $H$-maximal graphs some of them can be mutually isomorphic. So, we determine how many different isomorphism classes they belong to.

Now we give a description of a divisor concept. Given an $s \times s$ matrix $D=\left(d_{i j}\right)$, let the vertex set of a graph $G$ (of order $n$ ) be partitioned into non-empty subsets $V_{1}, V_{2}, \ldots, V_{s}$ so that for any $i, j \in\{1,2, \ldots, s\}$ each vertex from $V_{i}$ is adjacent to exactly $d_{i j}$ vertices of $V_{j}$. The multidigraph $F$ with adjacency matrix $D$ is called a front divisor of $G$, or briefly, a divisor of $G$ (see [6], Definition 2.4.4).

The eigenvalue $\lambda$ of graph $G$ is a main eigenvalue if and only if the corresponding eigenvector is not orthogonal to $(1,1, \ldots, 1)^{T}$ (compare [6], p. 25 and Theorem 2.2.3). Otherwise, $\lambda$ is said to be a non-main eigenvalue of $G$. The main part of the spectrum of $G$ consists of all its main eigenvalues.

It is known that the characteristic polynomial of a divisor divides the characteristic polynomial of a graph (cf. [6], p. 38). Moreover, due to Theorem 2.4.5 of [6], we have that the 


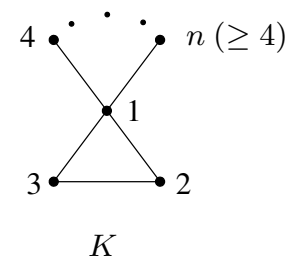

Figure 1: The infinite family of unicyclic star complements for 1 as the second largest eigenvalue (the vertices $4, \ldots, n$ form a totaly disconnected graph).

spectrum of any divisor $F$ of graph $G$ includes the main part of the spectrum of $G$.

We conclude this section by the following two definitions. The cocktail party graph with $2 k$ vertices, denoted by $C P_{k}$, is a complement of $k K_{2}$ (a regular graph of degree 1 , with $2 k$ vertices). An arbitrary connected graph is said to be $k$-cyclic if $m=n+k+1$ holds, where $n$ and $m$ are its order and size, respectively. For $k=0,1$ and 2 we get trees, unicyclic and bicyclic graphs, respectively.

\section{SCL - star complement library}

The SCL is free software which can be redistributed and/or modified under the terms of the GNU General Public License as published by the Free Software Foundation version 2.

There are three versions (written in $\mathrm{C}++$ ) of SCL: v. 1.0 was written by Zoran Stanić, while most of the code of v. 2.0 and 2.1 was written by Zoran Stanić and Nedeljko Stefanović (the only exception is the file cliques.cpp regarding the maximal clique search written by Kevin O'Neill - this part of the software one can redistribute and/or modify under the terms of the GNU General Public License as published by the Free Software Foundation; either version 2 of the License, or any later version). The SCL 1.0 is written for author's personal use. Namely, most of computational results from [13] and [14] are obtained by using it. There is just one difference between versions 2.0 and 2.1: v. 2.0 is a platform independent version with no GUI (graphical user interface), while v. 2.1 includes a GUI (developed by using QT OpenSource). SCL 2.0 and SCL 2.1 are available at www.matf.bg.ac.yu/ zstanic/scl.htm (the same link can be found at http://dmoz.org/Science/Math/Combinatorics/Software/ ).

The distribution of SCL 2.x includes an executable file, a complete source, Users Guide (including a description of the star complement technique) and a number of examples.

\section{Some star complements for 1 as the second largest eigenvalue}

\subsection{The unicyclic graphs}

In [13] and [14] the authors use the star complement technique to obtain some of the graphs whose second largest eigenvalue is equal to 1 . In the later paper all unicyclic star complements for 1 as the second largest eigenvalue are determined. Namely, there are nine such graphs plus one infinite family (see Figure 1). For each of nine finite graphs, the good vertices and the maximal extensions are determined, while the infinite family is left for future research. Here we complete this.

In the next theorem we identify all good sets for the infinite family of graphs depicted in Figure 1. 
Theorem 2. Let $K$ be the graph from Figure 1, and let $U$ be a subset of $V(K)$. Further, let $T_{n-5}$ denote a set of any $n-5$ of the terminal vertices of $K$, while $T$ denotes a set of all terminal vertices of $K$. The graph $K(U)$ is a good extension if and only if $U$ has one of the following forms:

1. $\{i\}(4 \leq i \leq n)$;

2. $\{1, i\}(4 \leq i \leq n)$;

3. $\{2,3\} \cup T_{n-5}$;

4. $\{2\} \cup T$ and $\{3\} \cup T$, with $n=7$;

5. $\{1,2\} \cup T$ and $\{1,3\} \cup T$, with $n=11$;

6. $\{2,3\}$, with $n=5$;

7. $\{1,2,3\}$, with $n=7$;

8. $\{2\}$ and $\{3\}$, with $n=7$;

9. $\{1,2\}$ and $\{1,3\}$, with $n=11$.

Proof Let $u, U$ and $K(U)$ be good with respect to 1 as the second eigenvalue.

Assume firstly that $u$ is adjacent to exactly $k$ of the terminal vertices of $K$, and let $l=$ $n-k-3$ (i.e. there are $l$ of the terminal vertices non-adjacent to $u$ ). Denote by $D$ the matrix of divisor of $K(U)$. Clearly, the vertices of $K(U)$ can be partitioned into five sets: $V_{1}=\{1\} ; V_{2}=\{2,3\} ; V_{3}$, containing $k$ terminal vertices adjacent to $u ; V_{4}$, containing $l$ terminal vertices non-adjacent to $u ; V_{5}=\{u\}$. Hence, $D$ has the following form

$$
D=\left(\begin{array}{ccccc}
0 & 2 & l & k & 0 \\
1 & 1 & 0 & 0 & 0 \\
1 & 0 & 0 & 0 & 1 \\
1 & 0 & 0 & 0 & 0 \\
0 & 0 & 0 & k & 0
\end{array}\right)
$$

Note that $K(U)$ contains two sets of duplicate vertices (they have the same open neighbourhood): (1) the terminal vertices of $K$ adjacent to $u$ and (2) the terminal vertices of $K$ non-adjacent to $u$, as well as exactly two, so called, coduplicate vertices (they are labelled by 2 and 3). Thus, $K(U)$ contains 0 as an eigenvalue of multiplicity at least $k+l-2$ and -1 as an eigenvalue of multiplicity at least 1 . In addition, all these eigenvalues belong to the non-main part of $K(U)$ spectrum (compare with [6]). Thus, the remaining five eigenvalues of $K(U)$ coincide with the eigenvalues of its divisor. Consequently, we have that 1 is an eigenvalue of $K(U)$ if and only if it is an eigenvalue of its divisor.

Clearly, 1 is an eigenvalue of $D$ if and only if $\operatorname{det}(I-D)=0$ holds. We get

$$
\operatorname{det}(I-D)=-2+2 k
$$

Hence, the good sets have the form $\{i\}(4 \leq i \leq n)$.

Assume now that $u$ is adjacent to the vertex labelled by 1 and to exactly $k$ of the terminal vertices of $K$. Using the notation and argument of the previous case we get 


$$
D=\left(\begin{array}{lllll}
0 & 2 & l & k & 1 \\
1 & 1 & 0 & 0 & 0 \\
1 & 0 & 0 & 0 & 1 \\
1 & 0 & 0 & 0 & 0 \\
1 & 0 & 0 & k & 0
\end{array}\right)
$$

and again

$$
\operatorname{det}(I-D)=-2+2 k \text {. }
$$

Hence, the good sets have the form $\{1, i\}(4 \leq i \leq n)$.

Similarly, if $u$ is adjacent to the vertices 2, 3 and to exactly $k$ of the terminal vertices of $K$, the good sets have the form $\{2,3\} \cup T_{n-5}$, where $T_{n-5}$ is specified above. If $u$ is adjacent to exactly one of the vertices 2,3 and to exactly $k$ of the terminal vertices of $K$, the good sets exist only if $n=7$, and then they have the form $\{2\} \cup T$, and $\{3\} \cup T$. The remaining cases are similar and will be omitted. ments.

Using the SCL, one can compute the maximal extensions for this family of star comple-

\subsection{The cocktail party graphs}

Here we consider the cocktail party graphs $C P_{k}$ as the star complements for 1 as the second largest eigenvalue. We prove the following theorem.

Theorem 3. Let $C P_{k}$ be the cocktail party graph of order $2 k$, and let $U$ be a subset of $V\left(C P_{k}\right)$. The graph $C P_{k}(U)$ is a good extension if and only if $U$ is:

1. a complement of any triangle of $C P_{k}$ or

2. any induced path of the length 2 of $C P_{6}$.

Proof Let $u, U$ and $C P_{k}(U)$ be good with respect to 1 as the second eigenvalue.

First we fix the neighborhood of $u$ : let $p$ pairs of non-adjacent vertices (in $C P_{k}$ ) are adjacent to $u$; there are $q$ pairs of non-adjacent vertices such that $u$ is adjacent to exactly one vertex of each pair, and there are $r$ pairs of non-adjacent vertices which are not adjacent to $u$. Clearly, $p+q+r=k$. As in Theorem 2, the matrix $D$ of the divisor $C P_{k}(U)$ has the following form

$$
D=\left(\begin{array}{ccccc}
2 p-2 & q & q & 2 r & 1 \\
2 p & q-1 & q-1 & 2 r & 1 \\
2 p & q-1 & q-1 & 2 r & 0 \\
2 p & q & q & 2 r-2 & 0 \\
2 p & q & 0 & 0 & 0
\end{array}\right)
$$

Since each eigenvalue of $C P_{k}(U)$ different from zero is an eigenvalue of its divisor, we get that 1 is an eigenvalue of $C P_{k}(U)$ if and only if $\operatorname{det}(I-D)=0$ holds. We compute

$$
\operatorname{det}(I-D)=3\left(9-6(2 p+2 q+r)+4(p q+p r+r q)+3 q^{2}\right)
$$


First, if $q$ is not odd then $\operatorname{det}(I-D)$ is odd. Further, if $q \geq 5$, we get $\operatorname{det}(I-D)>0$. Thus, $\operatorname{det}(I-D) \neq 0$ holds, whenever $q \notin\{1,3\}$. If $q=1$, we get $\operatorname{det}(I-D)=2(-4 p-r+$ $2 p r)=0$. Only integral non-negative solution is $(p, q, r)=(1,1,4)$ (for $(p, q, r)=(0,1,0)$ the graph considered reduces to $K_{2}$ in which 1 is the largest eigenvalue), i.e. $u$ is adjacent to any induced path of the length 2 of $C P_{6}$. For $q=3$, we get $\operatorname{det}(I-D)=2(3 r+2 p r)=0$. The infinite family of solutions is $(p, q, r)=(p, 3,0), p \geq 0$, i.e. $u$ is adjacent to the complement of any triangle of $C P_{k}$.

Let $u(T)$ and $u\left(T^{\prime}\right)$ be good vertices adjacent to the complements of triangles $T$ and $T^{\prime}$ of $C P_{k}$, respectively. Using the divisor concept we check that $u(T)$ and $u\left(T^{\prime}\right)$ are good partners in exactly two situations: (1) $T$ and $T^{\prime}$ have two vertices in common while the remaining two vertices are non-adjacent (here, $u(T)$ and $u\left(T^{\prime}\right)$ are non-adjacent) and (2) $T$ and $T^{\prime}$ have one vertex in common while the remaining four vertices form a quadrangle (here, $u(T)$ and $u\left(T^{\prime}\right)$ are adjacent). Thus, the possible extensions of $C P_{k}, k \geq 3, k \neq 6$, can be obtained by combining such good vertices. Much interesting case is $C P_{6}$ since there we have another type of good vertices (as it is proved in the previous theorem). This case is considered by using the facilities of SCL, and the result is given in the next theorem.

Theorem 4. There are exactly nine non-isomorphic maximal extensions of star complement $C P_{6}$. Each has 26 vertices and contain the following good sets (the vertices of $C P_{6}$ are labelled by $1,2, \ldots, 12$, such that the vertices $2 i-1$ and $2 i(i=1,2, \ldots 6)$ are non-adjacent $)$ : $\{1,2,3\},\{1,2,4\},\{3,4,5\},\{3,4,6\},\{1,5,6\},\{2,5,6\}$. In the list below we give the spectrum, followed by the complements of the remaining good sets.

1. $\left[-6.4999,-3^{4},-2.0660,-2^{4},-1.2435,1^{14}, 15.8094\right]\{8,10,12\},\{7,10,12\}$, $\{8,9,12\},\{7,9,12\},\{2,4,6\},\{1,4,6\},\{2,3,6\},\{1,3,6\}$

2. $\left[-6.3716,-3^{4},-2.2731,-2^{4},-1.2510,1^{14}, 15.8956\right]\{8,10,12\},\{7,10,12\}$, $\{8,9,12\},\{7,9,12\},\{1,4,6\},\{2,3,6\},\{1,3,5\},\{1,3,6\}$

3. $\left[-5.8856,-3^{5},-2^{4},-1.2626,1^{14}, 16.1482\right]\{8,10,12\},\{7,10,12\},\{8,9,12\}$, $\{7,9,12\},\{2,4,6\},\{1,3,6\},\{1,4,5\},\{2,3,5\}$

4. $\left[-6.4172,-3^{4},-2.0644,-2^{4},-1.4107,1^{14}, 15.8923\right]\{8,10,12\},\{7,10,12\}$, $\{8,9,12\},\{7,9,12\},\{1,4,6\},\{2,4,6\},\{1,3,6\},\{2,3,6\}$

5. $\left[-6.2877,-3^{4},-2.2690,-2^{4},-1.4213,1^{14}, 15.9780\right]\{8,9,12\},\{7,10,12\}$, $\{7,9,12\},\{7,9,11\},\{1,4,6\},\{2,3,6\},\{1,3,5\},\{1,3,6\}$

6. $\left[-5.7930,-3^{5},-2^{4},-1.4360,1^{14}, 16.2290\right]\{8,9,12\},\{7,10,12\},\{7,9,12\}$, $\{7,9,11\},\{2,4,6\},\{1,3,6\},\{1,4,5\},\{2,3,5\}$

7. $\left[-6.1355,-3^{4},-2^{6}, 1^{14}, 16.1355\right]\{8,10,12\},\{7,9,12\},\{7,10,11\},\{8,9,11\}$, $\{1,4,6\},\{2,4,6\},\{2,3,6\},\{1,3,6\}$

8. $\left[-6,-3^{4},-2.2195,-2^{5}, 1^{14}, 16.2195\right]\{8,10,12\},\{7,9,12\},\{7,10,11\}$, $\{8,9,11\},\{1,4,6\},\{2,3,6\},\{1,3,5\},\{1,3,6\}$

9. $\left[-5.4659,-3^{5},-2^{5}, 1^{14}, 16.4659\right]\{8,10,12\},\{7,9,12\},\{7,10,11\},\{8,9,11\}$, $\{2,4,6\},\{1,3,6\},\{1,4,5\},\{2,3,5\}$

\subsection{Connected graphs with up to five vertices}

Here we identify connected graphs with up to five vertices which can be star complements for 1 as the second largest eigenvalue. Some similar results can be found in [4]. By using the $\mathrm{SCL}$, we get the result summarized in the following theorem. 

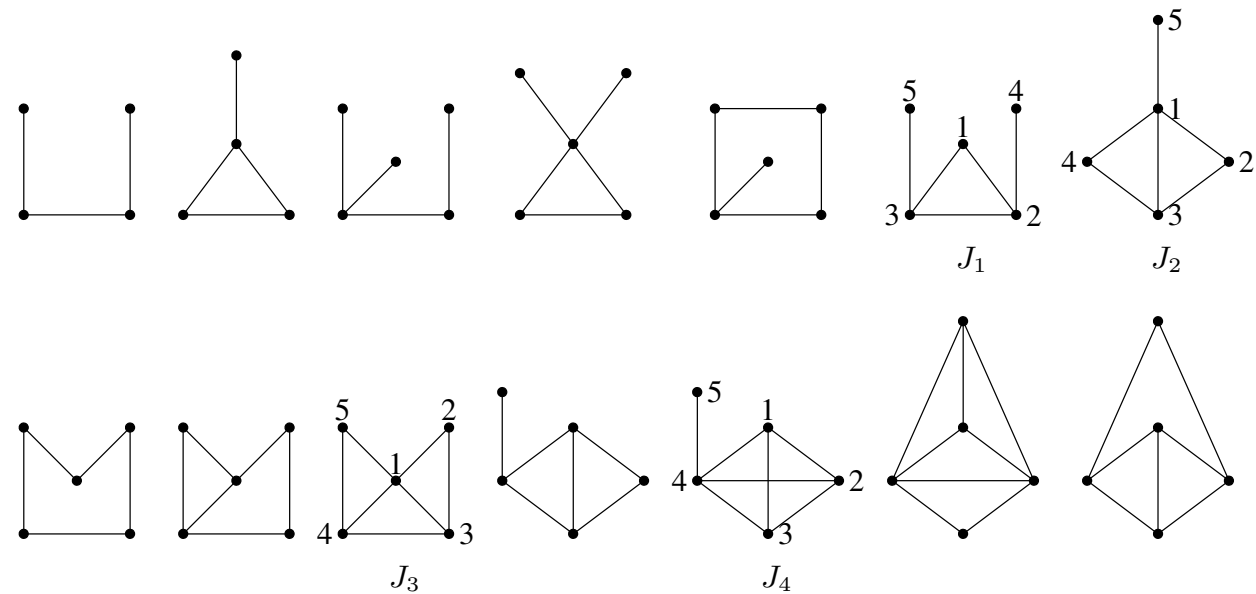

Figure 2: Connected star complements for 1 as the second largest eigenvalue with up to five vertices.

Theorem 5. There are exactly 14 connected graphs up to five vertices which can be star complements for 1 as the second largest eigenvalue. Those graphs are depicted in Figure 2.

As in the previous subsections, the maximal extensions can be easily computed. It is interesting that each of the graphs $J_{1}-J_{4}$ of Figure 2 has a unique maximal extension, i.e. all its good vertices are mutually compatible. In addition, the maximal extensions of $J_{2}$ and $J_{3}$ are isomorphic. In the list below, we present those extensions: the number of vertices, the spectrum and the good sets are given.

$$
\begin{aligned}
& J_{1}: 9\left[-2^{4}, 1^{4}, 4\right]\{1,4\},\{1,5\},\{2,4,5\},\{3,4,5\} \\
& J_{2}: 7\left[-2,-1.6458,-1^{2}, 1^{2}, 3.6458\right]\{1,2,5\},\{1,4,5\} \\
& J_{3}: 7\left[-2,-1.6458,-1^{2}, 1^{2}, 3.6458\right]\{1,2\},\{1,5\} \\
& J_{4}: 6[-2,-1.2361,-1,0,1,3.2361]\{1,5\}
\end{aligned}
$$

\section{Trees, unicyclic and bicyclic graphs as star complements for $\frac{\sqrt{5}-1}{2}$ as the second largest eigenvalue}

Let $\sigma=\frac{\sqrt{5}-1}{2}$ (the golden section). The graphs whose second largest eigenvalue does not exceed the golden section are intensively studied in, for example, [8], [9], [12], e.g., or [11] where a survey is given. Here we provide some results regarding the star complement technique.

Lemma 6. The path $P_{3}$ is the only tree, while the graphs in Figure 3 are the only unicyclic and bicyclic graphs which can be star complements for $\sigma$ as the second largest eigenvalue.

Proof Since $P_{4}$ contains $\sigma$ as its second largest eigenvalue, an arbitrary tree which is a star complement for $\sigma$ as the second largest eigenvalue cannot contain $P_{4}$ as an induced subgraph. Thus, such a tree must be a star. Now, let $S_{n}$ be a star of order $n$, and let $u, U$ and $S_{n}(U)$ be good with respect to $\sigma$. 
Assume first that $u$ is adjacent to exactly $k$ of the terminal vertices of $S_{n}$, and let $l=$ $n-k-1$ (i.e. there are $l$ terminal vertices not adjacent to $u$ ). Denote by $D$ the matrix of divisor of $S(U)$. As in Theorems 2 and 3, we get

$$
D=\left(\begin{array}{llll}
0 & l & k & 0 \\
1 & 0 & 0 & 0 \\
1 & 0 & 0 & 1 \\
0 & 0 & k & 0
\end{array}\right)
$$

and

$$
\operatorname{det}(\sigma I-D)=\sigma^{4}-(2 k+l) \sigma^{2}+k l .
$$

By putting $\sigma^{4}=2-3 \sigma$ and $\sigma^{2}=1-\sigma$ into the equation above, we get that $\operatorname{det}(\sigma I-D)=0$ holds only if $k=l=1$.

Assume now that $u$ is adjacent to the center of $S_{n}$ and to exactly $k$ of the terminal vertices of $S_{n}$. We compute

$$
D=\left(\begin{array}{llll}
0 & l & k & 1 \\
1 & 0 & 0 & 0 \\
1 & 0 & 0 & 1 \\
1 & 0 & k & 0
\end{array}\right)
$$

and

$$
\operatorname{det}(\sigma I-D)=\sigma^{4}-(2 k+l+1) \sigma^{2}-2 k \sigma+k l .
$$

It can be easy checked that the equation $\operatorname{det}(\sigma I-D)=0$ has no solutions in this case. Therefore, a path $P_{3}$ is the only tree which can be a star complement for $\sigma$ as the second largest eigenvalue.

Now, let us consider the unicyclic star complements. Since $C_{n}(n \geq 5)$ has second largest eigenvalue greater than $\sigma$, we should consider only the graphs which contain cycle $C_{3}$ or $C_{4}$. We find that $C_{3}, C_{4}$ and the graphs $L_{1}-L_{3}$ of Figure 3 are the only unicyclic graphs whose second largest eigenvalue does not exceed $\sigma$. In what remains we have to see which of these graphs afford good extensions for $\sigma$. Firstly, for $L_{1}-L_{3}$ we can find at least one good set (in addition, all good sets are given in the next theorem). Secondly, it is easy to check (say by a brute force computation using SCL) that the cycles mentioned do not contain any good set.

Finally, considering the bicyclic graphs and their spectra we check that $L_{4}-L_{6}$ are the only solutions.

The results obtained by making use of SCL facilities are given in the next theorem.

Theorem 7. There are exactly nine non-isomorphic maximal extensions of star complements $L_{1}-L_{4}$ of Figure 3. In the list below the following data are given: the number of vertices, the spectrum, followed by the good sets (the good sets of graphs $1-3$ (resp. $4-6$ and $7-$ 9) regard star complement $L_{1}$ (resp. $L_{5}$ and $\left.L_{6}\right)$ - the good sets correspond to the vertex labelling).

1. $6\left[-1.6180^{2},-0.4142,0.6180^{2}, 2.4142\right]\{2\},\{3\}$ 


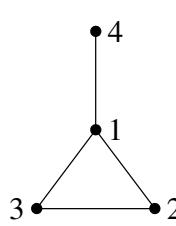

$L_{1}$

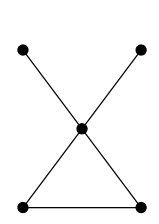

$L_{2}$

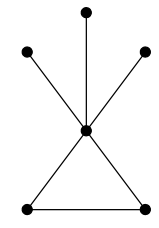

$L_{3}$

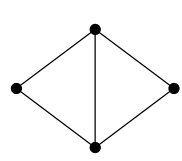

$L_{4}$

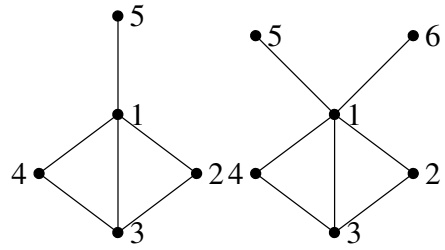

$L_{5}$

$L_{6}$

Figure 3: The unicyclic and bicyclic star complements for $\frac{\sqrt{5}-1}{2}$ as the second largest eigenvalue.

2. $6\left[-1.6180^{2},-1.2360,0.6180^{2}, 3.2360\right]\{2\},\{1,2,4\}$

3. $6\left[-1.6180^{2},-1.4995,0.6180^{2}, 3.4495\right]\{1,2,4\},\{1,3,4\}$

4. $7\left[-1.8434,-1.6180^{2}, 0.3068,0.6180^{2}, 3.53663\right]\{1,2\},\{1,4\}$

5. $7\left[-1.8284,-1.6180^{2}, 0,0.6180^{2}, 3.8284\right]\{1,4\},\{1,3,4,5\}$

6. $8\left[-1.8541,-1.6180^{3}, 0.6180^{3}, 4.8541\right]\{3\},\{1,2,3,5\},\{1,3,4,5\}$

7. $8\left[-2.1413,-1.6180^{2}, 0,0.5151,0.6180^{2}, 3.6262\right]\{1,2\},\{1,4\}$

8. $8\left[-2,-1.6180^{2},-1,0.4384,0.6180^{2}, 4.5616\right]\{1,2,3,6\},\{1,3,4,6\}$

9. $9\left[-2.1240,-1.6180^{3}, 0.3985,0.6180^{3}, 4.7255\right]\{1,4\},\{1,3,4,5\},\{1,3,4,6\}$

Remark 8. Note that $L_{2}$ is the star complement of graphs 4 and $5, L_{3}$ is the star complement of graphs 7 and 9 , while $L_{4}$ is the star complement of graphs $1-5$ and 8 .

\section{References}

[1] F. K. Bell and P. Rowlinson, On the multiplicities of graph eigenvalues, Bull. London Math. Soc. 35 (2003), 401-408.

[2] F. K. Bell and S. K. Simić, On graphs whose star complement for -2 is a path or a cycle, Linear Algebra Appl. 347 (2004), 249-265.

[3] D. Cvetković, M. Doob and H. Sachs, Spectra of graphs - theory and application, 3rd edition, Jonah Ambrosius Barth Verlag, Heidelberg-Leipzig, 1995.

[4] D. Cvetković, M. Lepović, P. Rowlinson and S. K. Simić, A database of star complements of graphs, Univ. Beograd, Publ. Elektrotehn. Fak., Ser. Mat. 9 (1998), 103-112.

[5] D. Cvetković and P. Rowlinson, Spectral graph theory, in: Lowell W. Beineke, Robin J. Wilson (eds.), Topics in algebraic graph theory, Cambridge University Press, 2004, 88-112.

[6] D. Cvetković, P. Rowlinson and S. Simić, Eigenspaces of graphs, Cambridge Univiversity Press, Cambridge, 1997.

[7] D. Cvetković, P. Rowlinson and S. Simić, Spectral generalizations of line graphs - on line graphs with least eigenvalue -2, Cambridge University Press, Cambridge, 2004.

[8] D. Cvetković and S. K. Simić, On graphs whose second largest eigenvalue does not exceed $\frac{\sqrt{5}-1}{2}$, Discrete Math. 138 (1995), 213-227.

[9] D. Cvetković and S. K. Simić, Minimal graphs whose second largest eigenvalue is not less than $\frac{\sqrt{5}-1}{2}$, Bull. Acad. Serbe. Sci. Math. 121 (2000), 47-70. 
[10] M. Ellingham, Basic subgraphs and graph spectra, Australasian J. Comb. 8 (1993), 247-265.

[11] M. Petrović and Z. Radosavljević, Spectrally constrained graphs, Faculty of Science, Kragujevac, Yugoslavia, 2001.

[12] S. K. Simić, Some notes on graphs whose second largest eigenvalue is less than $\frac{\sqrt{5}-1}{2}$, Linear and Multilin. Algebra 39 (1995), 59-71.

[13] Z. Stanić, On graphs whose second largest eigenvalue equals 1 - the star complement technique, Linear Algebra Appl. 420 (2007), 700-710.

[14] Z. Stanić and S. K. Simić, On graphs with unicyclic star complement for 1 as the second largest eigenvalue, in: Neda Bokan, Mirjana Djorić, Anatoly T. Fomenko, Zoran Rakić, Bernd Wegner, Julius Wess (eds.), Proceedings of the Conference contemporary geometry and related topics, Belgrade, June 26-July 2 2005, Faculty of Mathematics, Belgrade, 2006, 475-484. 